\title{
Phase with Spinel Structure Type in Plastically Deformed NiTi
}

\author{
Fedor M. Noskov*a, Liudmila I. Kveglis ${ }^{a}$, \\ Artur K. Abkaryan ${ }^{a}$ and Michail N. Volochaev ${ }^{b}$ \\ ${ }^{a}$ Siberian Federal University \\ 79 Svobodny, Krasnoyarsk, 660041, Russia \\ ${ }^{b}$ Kirensky Institute of Physics \\ 50/38 Akademgorodok, Krasnoyarsk, 660036, Russia
}

Received 27.07.2016, received in revised form 10.02.2017, accepted 29.04.2017

The alloy samples $\mathrm{Ni}_{51} \mathrm{Ti}_{49}$, subjected to plastic deformation were investigated. The microstructure and the microdiffraction were investigated by transmission electron microscopy Hitachi 7700. Discs with a diameter of $3 \mathrm{~mm}$ for investigation by transmission electron microscopy (TEM) was cut from the fracture zone of the stretched sample. They were mechanically thinned, then electrochemically etched until the hole in the center. The final step was to prepare ion etching in install PIPS (Gatan). TEM specimens were subjected cryomechanical processing. This was done by in liquid nitrogen cooling cyclically. The phase composition of the samples was determined by X-ray diffraction diffractometer "Bruker" using copper radiation. The lens-form crystals $\mathrm{Ni}_{2} \mathrm{Ti}_{3}$, containing bending contours, indicating significant internal stresses in the zones of stress localization were detected. The lens-form crystals can be represented as a non-equilibrium phase $\mathrm{Ni}_{2} \mathrm{Ti}_{3}$ with spinel structure type with lattice parameter $11,53 \pm 0,03 \mathrm{~A}$. For the formation of lenticular crystals of nonequilibrium phase $\mathrm{Ni}_{2} \mathrm{Ti}_{3}$ it is necessary redistribution of the original solid solution components. In local curvature of the crystalline lattice areas, the increased interatomic distances created the special structural states. These states increase the number of degrees of freedom in a deformable solid and thus contribute to the emergence of new phases.

Keywords: titanium nickelid, plastic deformation, lens-form crystals, spinel.

Citation: Noskov F.M., Kveglis L.I., Abkaryan A.K., Volochaev M.N. Phase with spinel structure type in plastically deformed niti, J. Sib. Fed. Univ. Eng. technol., 2017, 10(3), 292-304. DOI: 10.17516/1999-494X-2017-10-3-292-304.

(c) Siberian Federal University. All rights reserved

* Corresponding author E-mail address: yesoono@yandex.ru 


\title{
Фаза со структурным типом шпинели
}

\section{в пластически деформированном никелиде титана}

Ф.М. Носков ${ }^{a}$, Л.И. Квеглис ${ }^{a}$, А.К. Абкарян ${ }^{a}$, М.Н. Волочаев ${ }^{\sigma}$ ${ }^{a}$ Сибирский федеральныий университет Россия, 660041, Красноярск, пр. Свободный, 79 ${ }^{6}$ Институт физики СО РАН им. Л.В. Киренского Россия, 660036, Красноярск, Академгородок, 50/38

\begin{abstract}
Исследованы образиы сплава $N i_{51} T i_{49}$, подвергнутые пластической деформации. Микроструктура исследовалась методом просвечивающей электронной микроскопии и микродифракции на микроскопе Hitachi 7700. Для исследования образиов методами просвечивающей электронной микроскопии (ПЭМ) с зоны разрыва растянутых образиов вырезали диски диаметром 3 мм, которые механически утоняли, затем электрохимическим способом травили до появления отверстия в иентре диска. Финальным этапом подготовки являлось ионное травление на установке PIPS (Gatan). Утоненные для просвечивающей электронной микроскопии образиы были подвергнуты криомеханической обработке путем циклического охлаждения в жидком азоте. Фазовый состав образиов определяли методом дифракиии рентгеновских лучей в дифрактометре «Bruker» с использованием излучения меди. В зонах локализации деформации обнаружены линзовидные кристалльы фазы $\mathrm{Ni}_{2} \mathrm{Ti}_{3}$, содержащие изгибные экстинкиионные контуры, которые свидетельствуют о значительной кривизне кристаллической решетки, появляющейся в зонах локализачии пластической деформации. Показано, что кристаллическая структура линзовидных кристаллов представляет собой фазу, обладаюшую структурным типом шпинели с параметром

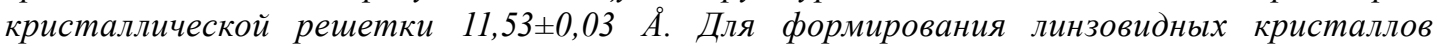
неравновесной фазы $\mathrm{Ni}_{2} \mathrm{Ti}_{3}$ необходимо перераспределение компонентов исходного твердого раствора или интерметаллидных фаз. В условиях локальной кривизны кристаллической решетки в зонах увеличенных межатомных расстояний возникают особые структурные состояния, которые повымают число степеней свободы в деформируемом твердом теле и таким образом способствуют появлению новых фаз.
\end{abstract}

Ключевые слова: никелид титана, пластическая деформация, линзовидные кристалль, шпинель.

Сплавы на основе никелида титана все более широко используются в качестве функциональных материалов в технике и медицине благодаря тому, что обладают набором уникальных физико-механических свойств [1-6].

Известно [7-10], что особенности мартенситного превращения в сплаве NiTi, такие как температура превращения, наличие и количество промежуточных фаз, носит ли превращение сверхпластичный характер или нет, очень зависит от наличия выделений $\mathrm{Ni}_{4} \mathrm{Ti}_{3}, \mathrm{Ti}_{2} \mathrm{Ni}$ и др. Выделения могут формироваться после термической обработки - закалки и отжига [2]. Исследования морфологии этих выделений показали, что выделения имеют линзовидную форму $[11,12]$. Они могут соответствовать как равновесным состояниям $\left(\mathrm{TiNi}_{3}, \mathrm{Ti}_{2} \mathrm{Ni}_{3}, \mathrm{TiNi}_{1} \mathrm{Ti}_{2} \mathrm{Ni}\right)$ [13], так и неравновесным $\left(\mathrm{Ti}_{11} \mathrm{Ni}_{14}\right)$ [14], $\left(\mathrm{Ti}_{3} \mathrm{Ni}_{4}\right)$ [15], причем последние могут достигать в размере 700 нм [12]. Кроме того, в структуре никелида титана могут присутствовать выделения равновесной оксидной фазы $\mathrm{Ti}_{4} \mathrm{Ni}_{2} \mathrm{O}[12,15]$. 
Главным фактором фазообразования при мартенситных превращениях в никелиде титана выступает коррелированность событий на разных масштабных уровнях [16]. Процессы перестройки кристаллической среды могут быть описаны как сложный иерархический процесс. Мартенситные превращения определяются элементарными актами кооперативного перемещения атомов и формированием специфической самоорганизованной мартенситной макроструктуры. В процессе формирования такой структуры может происходить образование выделений различных фаз.

В нашей работе [17] исследованы подвергнутые растяжению на разрывной машине утоненные образцы $\mathrm{Ni}_{51} \mathrm{Ti}_{49}$. Кончик разорванного образца подготавливали для исследования на просвечивающем электронном микроскопе по методике, описанной в [17]. Утоненные для просвечивающей электронной микроскопии образцы были подвергнуты циклическим мартенситным превращениям путем криомеханической обработки. На электронно-микроскопических изображениях наблюдалась система линзовидных кристаллов, сформированных напряжениями в области предразрушения на острие трещины. Такая система линзовидных кристаллов появлялась только после криомеханической обработки предварительно растянутых образцов, т.е. пластическая деформация, вызванная криомеханической обработкой, спровоцировала возникновение линзовидных кристаллов, пронизанных экстинкционными контурами. При исследовании химического состава линзовидных кристаллов с помощью энергодисперсионного метода [17] выявлено, что их химический состав соответствовал интерметаллиду $\mathrm{Ni}_{2} \mathrm{Ti}_{3}$. Исследование образцов, испытавших множественные циклы прямых и обратных мартенситных переходов, показало, что они обладают ненулевой намагниченностью, вызванной ростом новой ферромагнитной фазы [17].

Примеры получения фазы типа $\mathrm{Ni}_{2} \mathrm{Ti}_{3}$ известны. Так, в работе [18] такая фаза была получена при спекании с помощью электрического тока большой силы порошковых прессовок из чистых никеля и титана, взятых в равных пропорциях. В работе [19] выявлены несколько максимумов на картине рентгеновской дифракции, принадлежащих фазе $\mathrm{Ni}_{2} \mathrm{Ti}_{3}$. Однако структурный тип этой фазы не определен. В таблицах ASTM эта фаза также отсутствует.

В работе [20] порошки чистых титана и никеля, взятые в пропорции, соответствующей соединению $\mathrm{Ni}_{2} \mathrm{Ti}_{3}$, сплавляли индукционным методом. После этого сплав механически измельчали в порошок, который в свою очередь подвергали механической активации в барабанном активаторе с шарами в атмосфере аргона в течение 10 ч. Представленный в [20] спектр рентгеновской дифракции сплава после плавки в индукционной печи демонстрировал наличие двух фаз $\mathrm{NiTi}+\mathrm{Ti}_{2} \mathrm{Ni}$. Фаза $\mathrm{Ni}_{2} \mathrm{Ti}_{3}$ не наблюдалась. Спектр этого же сплава после механической активации характеризуется нанокристаллическим состоянием с высокой степенью дисперсности.

Авторы работы [21] получали сплав никелида титана из порошковой смеси при помощи ее пластической деформации взрывом. В результате образцы содержали, помимо матрицы со структурой В2 с мартенситами R и В19', некоторое количество линзовидных кристаллов $\mathrm{Ti}_{2} \mathrm{Ni}$, $\mathrm{Ti}_{3} \mathrm{Ni}_{4}, \mathrm{Ti}_{2} \mathrm{Ni}_{3}$ с внутренними неоднородностями.

Таким образом, исследование соединения $\mathrm{Ni}_{2} \mathrm{Ti}_{3}$ представляет собой научный и практический интерес.

Цель paботы: исследовать особенности структуры линзовидных кристаллов состава $\mathrm{Ni}_{2} \mathrm{Ti}_{3}$, формирующихся при пластической деформации образцов никелида титана. 
Задачи paботы: при помощи электронной микроскопии и рентгенофазового анализа исследовать структуру деформированных утоненных массивных образцов $\mathrm{Ni}_{51} \mathrm{Ti}_{49}$; методом дифракции электронов установить структурный тип формирующихся при деформации линзовидных кристаллов.

\section{Методика}

Исходные прутки сплава $\mathrm{Ni}_{51} \mathrm{Ti}_{49}$ подвергались прокатке в калибрах при температуре $800{ }^{\circ} \mathrm{C}$. Методом электроискровой резки вырезались образцы для механических испытаний на растяжение в форме двойных лопаток. Отжиг и закалка образцов, помещенных в запаянную кварцевую трубку, наполненную аргоном, проводились в камерной электропечи. Образцы помещались в печь после ее предварительного нагрева. Нагрев образцов проводился до температуры отжига $950 \pm 20^{\circ} \mathrm{C}$ с выдержкой в течение 1 ч и охлаждением до комнатной температуры в печи. Закалка отожженных образцов с температуры $850{ }^{\circ} \mathrm{C}$ с предварительной выдержкой в печи в течение 1 ч осуществлялась в воде. Образцы после металлографической обработки были подвергнуты статическому растяжению до разрыва. Растяжение образцов проводилось на электромеханической универсальной испытательной машине. Растянутые образцы в области шейки утонялись с помощью установки FIB. Для исследования образцов методами просвечивающей электронной микроскопии (ПЭМ) с зоны разрыва вырезали диски диаметром 3 мм, которые механически утоняли до толщины порядка 200 мкм, затем электрохимическим способом травили до появления отверстия в центре диска. Финальным этапом подготовки образцов служило ионное травление на установке PIPS (Gatan). Утоненные для просвечивающей электронной микроскопии образцы были подвергнуты криомеханической обработке путем циклического охлаждения в жидком азоте. Микроструктура исследовалась методом просвечивающей электронной микроскопии и микродифракции на микроскопе Hitachi 7700. Фазовый состав образцов определяли методом дифракции рентгеновских лучей в дифрактометре «Bruker» с использованием излучения меди.

\section{Экспериментальные результаты}

На рис. 1 представлен спектр рентгеновской дифракции, полученный от растянутого образца $\mathrm{Ni}_{51} \mathrm{Ti}_{49}$, подвергнутого криомеханической обработке. Линии фазы $\mathrm{Ni}_{2} \mathrm{Ti}_{3}$, взятые из [19], нанесены на экспериментальный спектр (рис. $1 a$, e) для идентификации. Видно присутствие фазы $\mathrm{Ni}_{2} \mathrm{Ti}_{3}$ в экспериментальном спектре. В спектре наблюдаются также линии от аустенита со структурой B2, мартенсита B19' (Карта ASTM №35-1281), соединений Ti ${ }_{2} \mathrm{Ni}$ (Карта ASTM №180898) и $\mathrm{Ti}_{3} \mathrm{Ni}_{4}$ (Карта ASTM №39-1113). Линии кислородной шпинели $\mathrm{Ni}_{2} \mathrm{Ti}_{4} \mathrm{O}$ (Карта ASTM №00-005-0694) с целью ее обнаружения нанесены на экспериментальный спектр на рис. 1ж. Фаза $\mathrm{Ni}_{2} \mathrm{Ti}_{4} \mathrm{O}$ не выявляется.

На рис. $2 a$ приведено электронно-микроскопическое изображение утоненного образца $\mathrm{Ni}_{51} \mathrm{Ti}_{49}$ в области шейки, растянутого до разрыва образца. Наблюдается линзовидный кристалл с экстинкционными контурами внутри, свидетельствующими о значительных внутренних напряжениях, возникающих в кристалле. Картина дифракции электронов, полученная с этого участка, показана на рис. 26. 

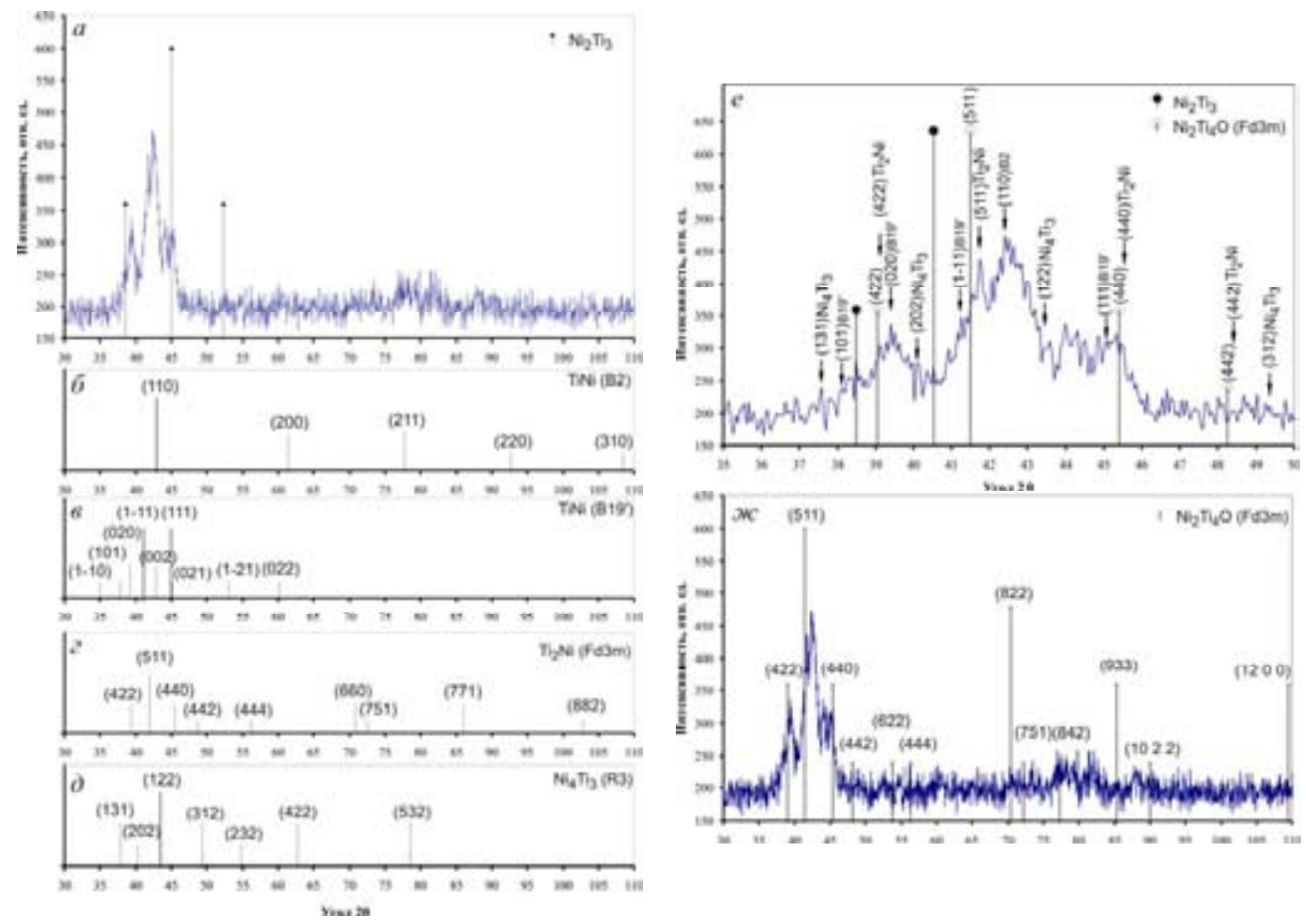

Рис. 1. Спектр рентгеновской дифракции от термически обработанного и растянутого образца $\mathrm{Ni}_{51} \mathrm{Ti}_{49}$, подвергнутого криомеханической обработке: $a$ - экспериментальный спектр, совмещенный с линиями соединения $\mathrm{Ni}_{2} \mathrm{Ti}_{3} ; \sigma$ - основные линии аустенита со структурой В2; в - основные линии мартенсита В19'; 2 - основные линии соединения $\mathrm{Ti}_{2} \mathrm{Ni} ; \partial$ - основные линии соединения $\mathrm{Ti}_{3} \mathrm{Ni}_{4} ; e$ - увеличенный фрагмент спектра; ж- экспериментальный спектр, совмещенный с линиями соединения $\mathrm{Ti}_{4} \mathrm{Ni}_{2} \mathrm{O}$

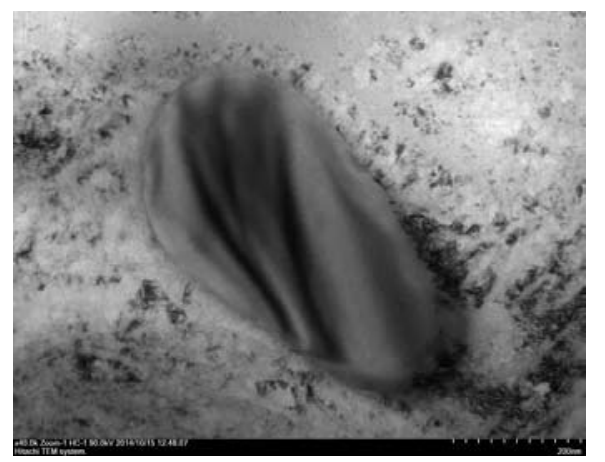

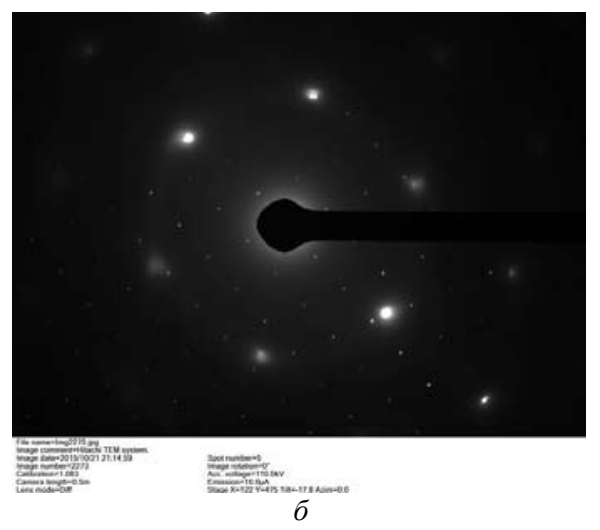

Рис. 2. Электронно-микроскопическое изображение с области шейки растянутого образца $\mathrm{Ni}_{51} \mathrm{Ti}_{49}(a)$ и дифракция электронов с этого участка (б)

Схема расшифровки полученной дифракционной картины показана на рис. 3, данные сведены в табл. 1.

Если ряд ярких рефлексов, лежащих на линии 9, принять за рефлексы [110] $]_{\mathrm{B} 2}$ с параметром 2,12 § (что соответствует параметру $3,01 \AA$ для рефлекса [100] ОЦК решетки В2 [6]), то 

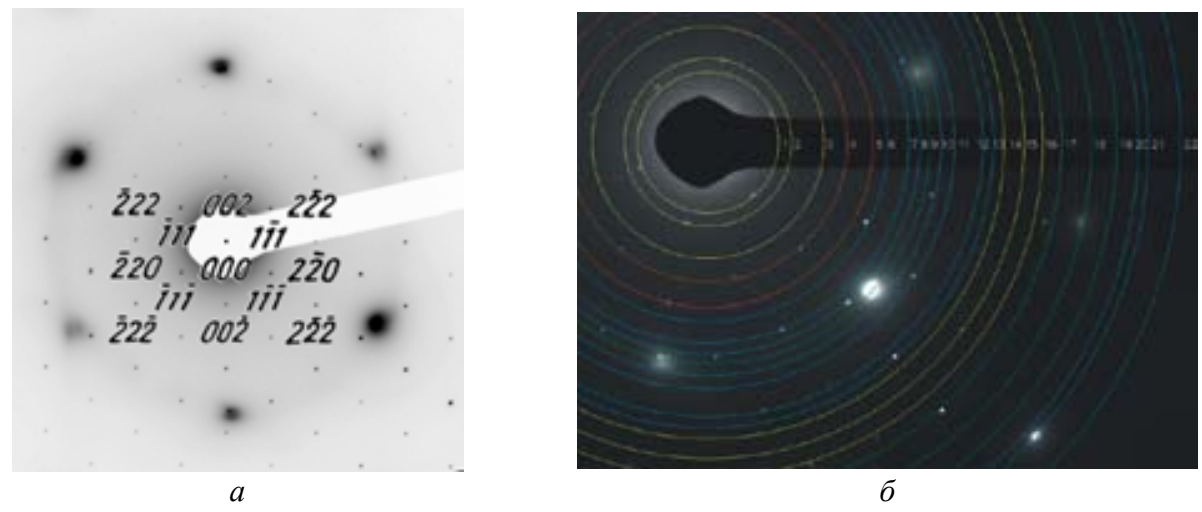

Рис. 3. Расшифровка картины дифракции электронов, полученная от участка с области шейки растянутого образца $\mathrm{Ni}_{51} \mathrm{Ti}_{49}: a$ - ориентация ГЦК [110]; $\sigma$ - схема расшифровки дифрактограммы, сведенная в табл. 1

Таблица 1. Результаты расшифровки дифракционной картины, приведенной на рис. 3

\begin{tabular}{|c|c|c|c|}
\hline $\begin{array}{l}\text { Номер } \\
\text { линии }\end{array}$ & $d, \AA$ & $\begin{array}{c}\text { Матрица В2, } \\
h k l(\Delta d, \AA)\end{array}$ & $\begin{array}{c}\text { Индексы структуры шпинели ориентации } \\
{[110], h k l(\Delta d, \AA)}\end{array}$ \\
\hline 1 & 6,65 & & $111(0,00)$ \\
\hline 2 & 5,61 & & $200(+0,15) ; 1 / 220(-0,03)$ \\
\hline 3 & 4,05 & & $220(+0,02)$ \\
\hline 4 & 3,38 & & $311(+0,11)$ \\
\hline 5 & 2,85 & & $400(+0,03)$ \\
\hline 6 & 2,62 & & $331(+0,02)$ \\
\hline 7 & 2,34 & & $422(+0,01)$ \\
\hline 8 & 2,22 & & $511(0,00)$ \\
\hline 9 & 2,12 & $110(0,00)$ & \\
\hline 10 & 2,01 & & $\mathbf{4 4 0}(+0,02)$ \\
\hline 11 & 1,91 & & $442(+0,01)$ \\
\hline 12 & 1,71 & & $533(+0,05)$ \\
\hline 13 & 1,65 & & $444(+0,01)$ \\
\hline 14 & 1,59 & & $551(+0,02)$ \\
\hline 15 & 1,49 & & $553(+0,01)$ \\
\hline 16 & 1,40 & & $644(0,00)$ \\
\hline 17 & 1,33 & & $751(0,00)$ \\
\hline 18 & 1,23 & $211(0,00)$ & $911(+0,03)$ \\
\hline 19 & 1,15 & & $933(+0,01)$ \\
\hline 20 & 1,09 & & $1040(-0,02)$ \\
\hline 21 & 1,07 & $220(-0,01)$ & $1042(-0,02)$ \\
\hline 22 & 0,99 & & $1060(0,00)$ \\
\hline
\end{tabular}

Примечание: 1. Числа в скобках показывают, сколько надо добавить/отнять от наших экспериментальных данных до идеального расчетного показателя.

2. Жирным шрифтом помечены основные рефлексы структуры шпинели. 
можно определить межплоскостные расстояния $d$ для всех линий (второй столбец табл. 1) [22]. Отсюда находим остальные рефлексы решетки В2, представленные в третьем столбце табл. 1.

Из рис. $3 a$ хорошо видно, что помимо фазы В2 в структуре сплава присутствует фаза с ГЦК решеткой ориентации [110]. Рабочей гипотезой была идея о возможности существования в структуре выделений сплава NiTi фазы, обладающей структурным типом шпинели. Поскольку шпинель имеет симметрию ГЦК решетки, то, исходя из того, что первая линия дифрактограммы (рис. 3б) с межплоскостным расстоянием, равным 6,65 А̊ (табл. 1), соответствует рефлексу [111] ГЦК-решетки (рис. 3a), можно определить параметр кристаллической решетки шпинели [22]. Так, если $d_{111}$ решетки ГЦК равен $6,65 \AA$, то параметр решетки шпинели $a_{100}=6,65 \times \sqrt{3}=11,51$ $\AA$. Зная параметр решетки шпинели, можно рассчитать $h k l$ для различных значений межплоскостных расстояний (последний столбец табл. 1).

На рис. 4 приведено электронно-микроскопическое изображение утоненного образца $\mathrm{Ni}_{51} \mathrm{Ti}_{49}$ в области шейки, растянутого до разрыва образца, и картина дифракции электронов от этого участка.

Схема расшифровки полученной дифракционной картины показана на рис. 4, данные сведены в табл. 2.

Методика расчета аналогична предыдущему случаю. Если ряд ярких рефлексов, лежащих на линии 7 (рис. 5б), принять за рефлексы [110] $]_{\text {в2 }}$ с параметром 2,12 $\AA$, то можно определить межплоскостные расстояния $d$ для всех линий (второй столбец табл. 2). Отсюда находим остальные рефлексы решетки В2, представленные в третьем столбце табл. 2.

Из рис. $5 a$ видно, что помимо фазы В2 в структуре сплава присутствует фаза с ГЦКрешеткой ориентации [211]. Исходя из того, что первая линия дифрактограммы (рис. 4б) с межплоскостным расстоянием, равным 6,67 $($ (табл. 1), соответствует рефлексу [111] ГЦК-решетки (рис. $5 a$ ), можно определить параметр кристаллической решетки шпинели. Так, если $d_{111}$ решетки ГЦК равен 6,67 $\AA$, то параметр решетки шпинели $a_{100}=6,67 \times \sqrt{3}=11,55 \AA$. Зная параметр решетки шпинели, можно рассчитать $h k l$ для различных значений межплоскостных расстояний (последний столбец табл. 2).

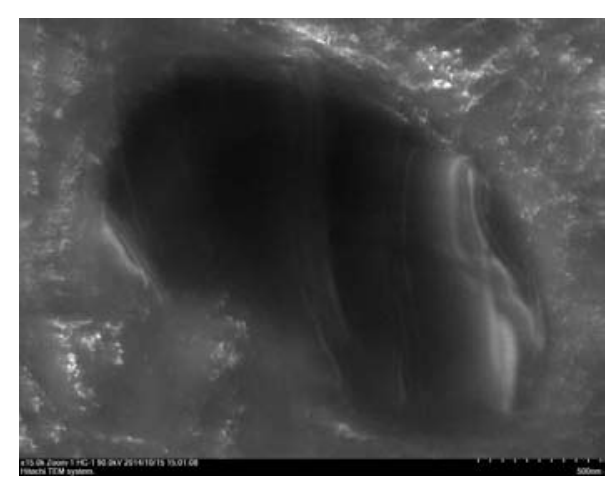

$a$

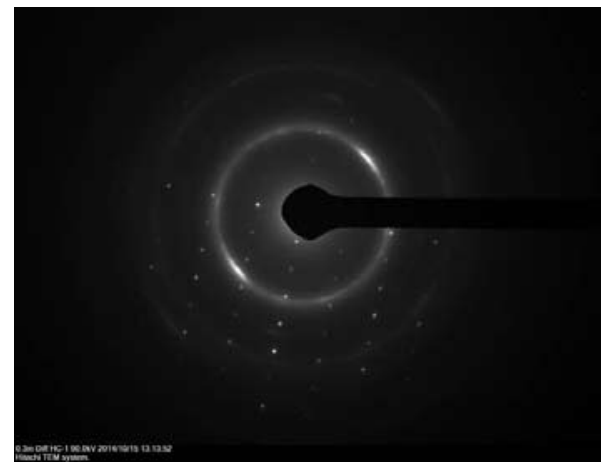

6

Рис. 4. Электронно-микроскопическое изображение с области шейки растянутого образца $\mathrm{Ni}_{51} \mathrm{Ti}_{49}$ в режиме темного поля $(a)$ и дифракция электронов с этого участка (б) 


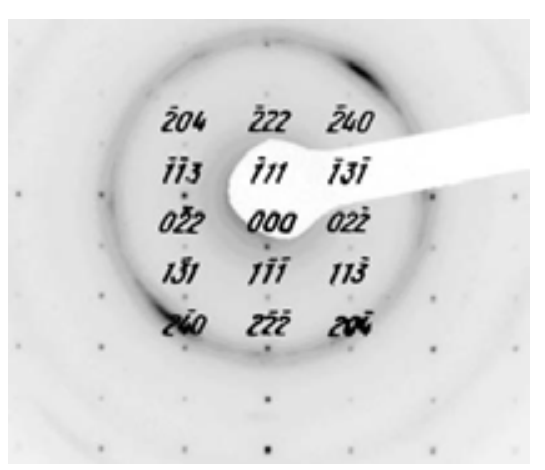

$a$

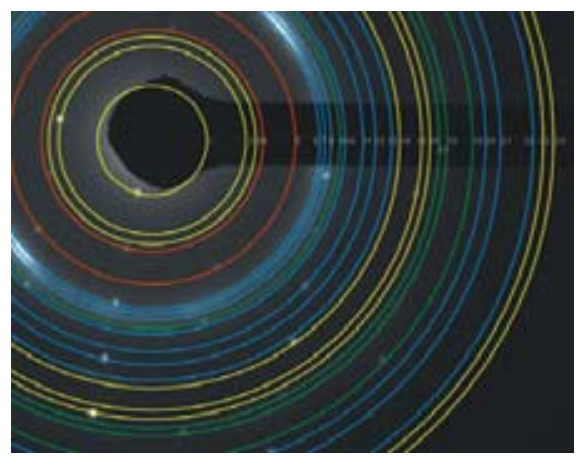

$\sigma$

Рис. 5. Расшифровка картины дифракции электронов, полученная от участка с области шейки растянутого образца $\mathrm{Ni}_{51} \mathrm{Ti}_{49}: a$ - ориентация ГЦК [211]; $\sigma$ - схема расшифровки дифрактограммы, сведенная в табл. 2

Таблица 2. Результаты расшифровки дифракционной картины, приведенной на рис. 5

\begin{tabular}{|c|c|c|c|}
\hline $\begin{array}{l}\text { Номер } \\
\text { линии }\end{array}$ & $d, \AA$ & $\begin{array}{c}\text { Матрица В2, } \\
h k l(\Delta d, \AA)\end{array}$ & $\begin{array}{c}\text { Индексы структуры шпинели ориентации } \\
{[211], h k l(\Delta d, \AA)}\end{array}$ \\
\hline 1 & 6,67 & & $111(0,00)$ \\
\hline 2 & 3,91 & & $220(+0,17) ; 221(-0,06)$ \\
\hline 3 & 3,53 & & $311(-0,05)$ \\
\hline 4 & 3,31 & & $222(+0,02)$ \\
\hline 5 & 2,56 & & $331(+0,09)$ \\
\hline 6 & 2,19 & & $511(+0,03)$ \\
\hline 7 & 2,12 & $110(0,00)$ & \\
\hline 8 & 2,04 & & $440(0,00)$ \\
\hline 9 & 1,95 & & $531(0,00)$ \\
\hline 10 & 1,90 & & $442(-0,03)$ \\
\hline 11 & 1,74 & & $\mathbf{5 3 3}(+0,02)$ \\
\hline 12 & 1,64 & & $444(+0,03)$ \\
\hline 13 & 1,53 & & $642(+0,01)$ \\
\hline 14 & 1,48 & $200(+0,03)$ & \\
\hline 15 & 1,36 & & $660(0,00)$ \\
\hline 16 & 1,32 & & $751(+0,01)$ \\
\hline 17 & 1,27 & & $911(0,00)$ \\
\hline 18 & 1,24 & $211(-0,01)$ & \\
\hline 19 & 1,14 & & $933(+0,02)$ \\
\hline 20 & 1,10 & & $1040(-0,03)$ \\
\hline 21 & 1,05 & $220(+0,01)$ & \\
\hline 22 & 0,96 & & $971(+0,06)$ \\
\hline 23 & 0,94 & & $1060(+0,05)$ \\
\hline 24 & 0,91 & & $1062(+0,07)$ \\
\hline
\end{tabular}

Примечание: 1. Числа в скобках показывают, сколько надо добавить/отнять от наших экспериментальных данных до идеального расчетного показателя.

2. Жирным шрифтом помечены основные рефлексы структуры шпинели. 


\section{Обсуждение результатов}

Из анализа результатов, полученных при расшифровке картин электронной дифракции от линзовидных кристаллов состава $\mathrm{Ni}_{2} \mathrm{Ti}_{3}$ (табл. 1, 2), следует, что в структуре исследованных сплавов помимо матричного твердого раствора со структурой В2 присутствует фаза со структурным типом шпинели. У шпинелей существует набор самых интенсивных рефлексов (показанных жирным шрифтом в табл. 1, 2), которые обычно используются для идентификации в справочной литературе, например в [22]. В нашем эксперименте выявлено бо́льшее количество рефлексов, отвечающих симметрии Fd3m. Большо́й параметр кристаллической решетки сам по себе говорит о том, что элементарная ячейка, имеющая структуру типа Fd3m, содержит внутри себя дополнительные атомы или имеет внутреннюю структуру. Параметр кристаллической

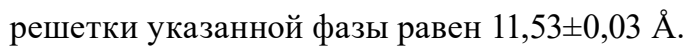

Структурный тип шпинели для интерметаллических соединений никеля и титана известен. В таблице ASTM фазы Ti 2 Ni (Карта ASTM №18-0898) [23] показано, что фаза имеет структурный тип Fd3m (шпинель) и параметр кристаллической решетки, равный 11,27.

Спектр рентгеновской дифракции рис. 1 демонстрирует наличие большого количества сосуществующих фаз, каждой из которых отвечает набор локальных рефлексов. Среди расшифрованных фаз наблюдается и аустенит, и мартенсит, и интерметаллидные фазы. Среди них имеется и система рефлексов от фазы $\mathrm{Ni}_{2} \mathrm{Ti}_{3}$ соответствующих табл. 1, 2. Здесь следует подчеркнуть тот факт, что рентгеновский спектр является интегральной характеристикой исследованного образца, а спектры дифракции электронов были сняты с конкретных локальных участков, характеризующихся наличием линзовидных кристаллов (рис. 2 и 4).

Рентгеновские исследования (рис. 1) демонстрируют отсутствие в структуре изученного образца кислородной шпинели $\mathrm{Ti}_{4} \mathrm{Ni}_{2} \mathrm{O}$ в сколько-нибудь значительных количествах, что в сочетании с показаниями энергодисперсионных спектров [17], свидетельствующих об отсутствии кислорода в исследованных линзовидных кристаллах, позволяет уверенно исключить то, что указанные кристаллы представляют собой фазу $\mathrm{Ti}_{4} \mathrm{Ni}_{2} \mathrm{O}$. Дополнительным свидетельством является разница параметров кристаллической решетки, которая для кислородных шпинелей в никелиде титана варьируется в пределах 11,27-11,35 А, что несколько меньше, чем полученная в нашем эксперименте $-11,53 \pm 0,03 \AA$.

Анализ табл. 1, 2 показывает, что практически смещение дифракционных рефлексов от расчетных значений не превышает $0,15 \AA$. Смещение линий дифрактограммы в линзовидных кристаллах, испытывающих кривизну, характеризующуюся изгибными экстинкционными контурами (см. рис. 2, 4), достаточно хорошо исследовано в работах В.Ю. Колосова, например в [24]. Искривлением кристаллических решеток можно частично объяснить несовпадение межплоскостных расстояний $d$ соответствующих рефлексов при сравнении табл. 1 и 2 . Возможность смещения атомов при мартенситных переходах экспериментально показана в работе [25], где атомы могут смещаться из своих позиций на величину до $0,2 \AA$. В работах В.Г. Пушина, например [26], показана возможность значительно бо́льших атомных смещений в никелиде титана.

Существуют отличия во внутренней морфологии равновесных и неравновесных фаз при их наблюдении в электронном микроскопе. Так, фаза $\mathrm{Ti}_{4} \mathrm{Ni}_{2} \mathrm{O}$, по данным [15], характеризуется однородной внутренней структурой, а фаза $\mathrm{Ti}_{3} \mathrm{Ni}_{4}$ в сплаве $\mathrm{Ni}_{50,8} \mathrm{Ti}_{49,2}$, например, в [12] характе-

$$
-300-
$$


ризуется наличием четко различимых экстинкционных контуров, говорящих о значительных внутренних напряжениях.

Появление изгибных контуров может быть объяснено на основе расчетов локальных напряжений в зоне предразрушения, сделанных в работе [27]. Однако если в работе [27] в 3оне предразрушения описывается возникновение трещины линзовидной формы, то в нашей работе [17] показано, что в зоне предразрушения (на острие трещины) возникают линзовидные кристаллы с изгибными контурами внутри. Таким образом, локализация деформации в нашем случае приводит к возникновению «промежуточного» состояния между состоянием предразрушения и разрушением - формированием неравновесной фазы с морфологией линзовидного кристалла со значительными внутренними напряжениями [17].

Очевидно, что для формирования линзовидных кристаллов неравновесной фазы $\mathrm{Ni}_{2} \mathrm{Ti}_{3}$ необходимо перераспределение компонентов исходного твердого раствора или интерметаллидных фаз. В условиях локальной кривизны кристаллической решетки в зонах увеличенных межатомных расстояний возникают особые структурные состояния, которые повышают число степеней свободы в деформируемом твердом теле. В работе [28] такие состояния были названы межузельными бифуркационными структурными состояниями (МБСС). Благодаря возникновению МБСС в деформированном металле возможна реализация направленного массопереноса, протекающего со скоростью переключения межатомных связей, которая в некоторых случаях может достигать скорости звука в металле [29].

В работе В.С. Крапошина [30] представлены примеры совместного (сопряженного) роста кристаллов разных фаз. Автор указывает, что причины сопряжения кристаллов до сих пор до конца не ясны. Принципиальная причина такой неудачи - то, что обычно сопоставляются геометрически подобные плоские сечения двух кристаллических структур, а кристаллическая структура по определению трехмерна и является результатом взаимодействия кристаллохимически допустимых (энергетически выгодных) строительных единиц, например координационных полиэдров. В работе показано, в частности, что шпинель может быть представлена как пример сращивания двух спиралей полиэдров с равными ребрами, но образованных ионами разной химической природы. Подход, основанный на анализе процессов структурообразования как взаимодействия координационных полиэдров, весьма перспективен.

\section{Выводы}

1. В образцах никелида титана, подвергнутых пластической деформации, обнаружены линзовидные кристаллы фазы $\mathrm{Ni}_{2} \mathrm{Ti}_{3}$, содержащие изгибные контуры, свидетельствующие о значительных внутренних напряжениях в локализованных областях.

2. Кристаллическая структура линзовидных кристаллов представляет собой фазу, обла-

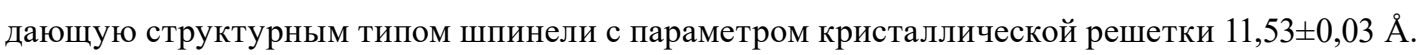

\section{Список литературы}

[1] Лихачев В.А., Кузьмин С.Л., Каменцова 3.П. Эффект памяти формыл. Л.: ЛГУ, 1987. 216 c. [Likhachev V.A., Kuz'min S.L., Kamentsova Z.P. The shape memory effect. Leningrad, LGU, 1987, 216 p. (in Russian)]

$$
\text { - } 301-
$$


[2] Гюнтер В.Э., Ходоренко В.Н., Ясенчук Ю.Ф. и др. Никелид титана. Медицинский материал нового поколения. Томск: МИЦ, 2006. 296 с. [Gyunter V.E., Khodorenko V.N., Yasenchuk Yu.F. and etc. Nickel titanium. Medical material of the new generation. Tomsk, MITs, 2006, 296 p. (in Russian)]

[3] Малыгин Г.А. Размытые мартенситные переходы и пластичность кристаллов с эффектом памяти формы. УФН, 2001, 171(2), 187-212 [Malygin G.A. Blurred martensitic transitions and plasticity of crystals with shape memory effect. UFN, 2001, 171(2), 187-212 (in Russian)]

[4] Потекаев А.И., Клопотов А.А. Козлов Э.В. и др. Слабоустойчивые предпереходные структуры в никелиде титана. Томск: НТЛ, 2004. 296 с. [Potekaev A.I., Klopotov A.A. Kozlov E.V. and etc. Weak-resistant pre-transition structures in titanium nickelide. Tomsk, NTL, 2004, 296 p. (in Russian)]

[5] Otsuka K., Ren X. Physical metallurgy of Ti-Ni-based shape memory alloys. Progr. Mat. Sci., 2005, 50, 511-678.

[6] Ооцука К, Симидзу К., Судзуки Ю. и др. Сплавы с эффектом памяти формы. Пер. с японск. М.: Металлургия, 1990. 224 с. [Otsuka K, Simidzu K., Sudzuki Yu. and etc. Alloys with shape memory effect. Moscow, Metallurgiya, 1990, 224 p. (in Russian)]

[7] Zel'dovich V., Sobyanina G., Novoselova T.V. Martensitic transformations in TiNi alloys with $\mathrm{Ti}_{3} \mathrm{Ni}_{4}$ precipitates. J. Phys, 1997, IV, 7(C5), 299-304.

[8] Bataillard L., Bidaux J.-E., Gotthardt R. Interaction between microstructure and multiplestep transformation in binary NiTi alloys using in-situ transmission electron microscopy observations. Philosophical Magazine A, 1998, 78(2), 327-344.

[9] Khalil-Allafi, J., Dlouhy, A. and Eggeler, G. $\mathrm{Ni}_{4} \mathrm{Ti}_{3}$-precipitation during aging of NiTi shape memory alloys and its influence on martensite phase transformation. Acta Materialia, 2002, 50, 4255-4274.

[10] Filip, P., Mazanec, K. On precipitation kinetics in TiNi shape memoryalloys. Scr Mater., 2001, 45, 701-707.

[11] Li Z.Q., Sun Q.P. The initiation and growth of macroscopic martensite band in nano-grained NiTi microtube under tension. International Journal of Plasticity, 2002, 18, 1481-1498.

[12] Efstathiou C., Sehitoglu H. Local transformation strain measurements in precipitated NiTi single crystals. Scripta Materialia, 2008, 59, 1263-1266.

[13] Клопотов А.А., Гюнтер В.Э., Марченко Е.С., Ясенчук Ю.Ф., Клопотов В.Д., Козлов Э.В. Кристаллогеометрия структур в системах Ti-Ni, Ti-Fe и Ti-Ni-Fe. Фундаментальные проблемь современного материаловедения, 2009, 6(4), 81-90 [Klopotov A.A., Gyunter V.E., Marchenko E.S., Yasenchuk Yu.F., Klopotov V.D., Kozlov E.V. Crystallogometry of structures in Ti-Ni, Ti-Fe, and Ti-Ni-Fe systems. Fundamental problems of modern materials science, 2009, 6(4), 81-90 (in Russian)]

[14] Tadaki T., Nakata Y., Shimizu K., Otsuka K. Crystal Structure, Composition and Morphology of a Precipitate in an aged Ti-51at.\%Ni Shape Memory Alloy. Trans. JIM, 1986, 27(10), 731-740.

[15] Сурикова Н.С. Закономерности имеханизмы пластической деформации и структурнофазовых превращений в монокристаллах сплавов $\mathrm{TiNi}(\mathrm{Fe}, \mathrm{Mo})$ и $\mathrm{TiNi}(\mathrm{Fe})$ : дис. ... д. ф.-м. н. : 01.04.07. Томск, 2011. 343 c. [Surikova N.S. Regularities and mechanisms of plastic deformation and 
structural-phase transformations in single crystals of alloys TiNi ( $\mathrm{Fe}, \mathrm{Mo}$ ) and TiNi (Fe), $\mathrm{PhD}$ thesis of specialty 01.04.07. Tomsk, 2011, 343 p. (in Russian)]

[16] Плотников В.А. Акустическая диссипащия энергии при термоупругих мартенситных превращениях в сплавах на основе никелида титана. Барнаул. Изд-во Алт. ун-та, 2013. 204 с. [Plotnikov V.A. Acoustic energy dissipation in thermoelastic martensitic transformations in alloys based on titanium nickelide. Barnaul. Publishing house Alt. University, 2013. 204 p. (in Russian)]

[17] Джес А.В., Носков Ф.М., Квеглис Л.И., Волочаев М.Н., Тажибаева Г.Б. Особенности формирования линзовидных кристаллов при мартенситных превращениях в никелиде титана. Фундаментальные проблемы современного материаловедения, 2016, 13(1), 96-104. [Dzhes A.V., Noskov F.M., Kveglis L.I., Volochaev M.N., Tazhibaeva G.B. Features of the formation of lenticular crystals in martensitic transformations in titanium nickelide. Fundamental problems of modern materials science, 2016, 13(1), 96-104 p. (in Russian)]

[18] Yener T., Siddique S., Walther F., Zeytin S. Effect of electric current on the production of NiTi intermetallics via electric-current-activated sintering. MTAEC9 Materials and technology, 2015, 49(5), 721-724.

[19] Ergin N., Ozdemir O. An investigation on TiNi intermetallic produced by electric current activated sintering. Acta Physica Polonica A, 2013, 123(2), 248-249.

[20] Xinxin C., Liqun M., Meng Y., Xiangyu Z., Yi D. Electrochemical Properties of the Amorphous $\mathrm{Ti}_{3} \mathrm{Ni}_{2}$ Alloy in Ni/MH Batteries. Rare Metal Materials and Engineering, 2012, 41(9), 1511-1515.

[21] Han X., Zou W., Wang R., Jin S., Zhang Z., Li T., Yang D. Microstructure of TiNi shapememory alloy synthesized by explosive shock-wave compression of Ti-Ni powder mixture. Journal of materials science, 1997, 32, 4723-4729.

[22] Горелик С.С., Скаков Ю.А., Расторгуев Л.Н. Рентгенографический и электроннооптический анализ М.:МИСИС, 1994. 328 с. [Gorelik S.S., Skakov Yu.A., Rastorguev L.N. X-ray and electron-optical analysis. Moscow, MISIS, 1994, 328 p. (in Russian)]

[23] Yurko G.A., Barton J.W., Parr J.G. The crystal structure of Ti2Ni. Acta Cryst., 1959, 12, 909911.

[24] Kolosov V.Yu. Tholen A.R. Transmission electron microscopy studies of the specific structure of crystals formed by phase transition in iron oxide amorphous films. Acta Materialia, 2000, 48, 1829.

[25] Lipson H., Parker A.M.B. Structure of Martensite. Jour. Iron Steel Inst., 1944, 149, $123-$ 141.

[26] Пушин В.Г., Кондратьев В.В., Хачин В.Н. Предпереходные явления и мартенситные превращения. Екатеринбург. УрО РАН, 1998. 367 с. [Pushin V.G., Kondrat'ev V.V., Khachin V.N. Pretransitional phenomena and martensitic transformations. Ekaterinburg, UB RAS, 1998, 367 p. (in Russian)]

[27] Корнев В.М. О диаграммах разрушения тел с короткими макротрещинами. Охрупчивание материала при усталостном разрушении. Физ. мезомех., 2016, 19(2), 80-99 [Kornev V.M. About diagrams of destruction of bodies with short macrocracks. Embrittlement of the material during fatigue failure. Phys. Mesomech., 2016, 19(2), 80-99 (in Russian)]

[28] Панин В.Е., Егорушкин В.Е. Основы физической мезомеханики пластической деформации и разрушения твердых тел как нелинейных иерархически организованных систем. Физ.

$$
-303-
$$


мезомех., 2015, 18(5), 100-113 [Panin V.E., Egorushkin V.E. Fundamentals of physical mesomechanics of plastic deformation and destruction of solids as nonlinear hierarchically organized systems. Phys. Mesomech., 2015, 18(5), 100-113 (in Russian)]

[29] Abylkalykova R.B., Kveglis L.I., Kalitova A.A., Noskov F.M. Abnormally fast migration of substance at shock loadings. Advanced Materials Research, 2014, 871, 231-234.

[30] Крапошин В.С., Талис А.Л., Демина Е.Д., Зайцев А.И. Кристаллогеометрический механизм срастания шпинели и сульфида марганца в комплексное неметаллическое включение. Металловедение и термическая обработка металлов, 2015, 721(7), 4-12 [Kraposhin V.S., Talis A.L., Demina E.D., Zaytsev A.I. The crystal-geometric mechanism of the merging of manganese spinel and sulphide into a complex nonmetallic inclusion. Metallurgy and heat treatment of metals, 2015, 721(7), 4-12] 